\title{
ENTRE NUESTROS OJOS Y EL MUNDO: ASIMILACIÓN DEL CONCEPTO DE ESPACIO EN EL RELATO "WALKER BROTHERS COWBOY” DE ALICE MUNRO
}

\author{
María Jesús Hernáez Lerena \\ Universidad de La Rioja
}

\begin{abstract}
RESUMEN: Este artículo establece un paralelismo entre un modelo de comprensión retórico, el propiciado por la metáfora, y un modelo de progresión en una trama narrativa, basado en la ruptura de las relaciones de cotidianidad entre el personaje y su entorno espacial. El relato "Walker Brothers Cowboy" no se estructura alrededor de una secuencia de sucesos, ni en torno a la modificación del carácter, ni siquiera se dirige a una revelación final, sino que apunta al rechazo de un esquema asentado de conocimiento: la solidez de la realidad externa, la estabilidad del escenario que rodea a la protagonista.

ABSTRACT: Metaphor produces new ways of access to the world. Fiction engages us in an adventure through time and space where characters readjust their relationships to other characters and to objects. This article draws a parallelism between the perceptual processes triggered off by metaphor and by plot in order to explore the ways in which this short story creates disturbance in the main character's anchorage to her world once she perceives that the essence of everyday reality, identified with a familiar landscape, will always evade her.
\end{abstract}

Yes, I use bits of what is real, in the sense of being really there and really happening, in the world, as most people see it, and I transform it into something that is really there and really happening, in my story.

Alice Munro, "What is Real?"

El hecho de que una parte de la opinión crítica canadiense reconociera una cualidad realista en las ficciones de Alice Munro contribuyó poderosamente a la infravaloración inicial de su obra. La aparente semejanza entre los relatos escritos por Munro y su propia vida y entorno geográfico (el Suroeste de Ontario) hizo que ciertos críticos clasificaran su obra como mimética, como un ejemplo más de la tradición realista canadiense que trata de personajes ordinarios atrapados en el restringido mundo de una comunidad aislada1. George Woodcock (1987:138), por ejemplo, en su capítulo

1. W. R. Martin (1987:99) y David Stouck (1988:260) consideran que los primeros relatos de Alice Munro, en los que se describían las experiencias de su niñez en un escenario canadiense reconocible, son 
sobre Alice Munro titulado “The Plots of Life: The Realism of Alice Munro" afirma que sólo hay dos formas de leer a Munro:

the exoteric one of the reader who knows a good story when he comes upon it and reads it with enjoyment and not too much concern for authenticity, and the esoteric one of the Canadian who is likely to read it with a special sense of its truth to the life he knows.

En mi opinión, esta segunda lectura que, según Woodcock, se basa en el reconocimiento de un espacio y de unas gentes reales, no se encuentra necesariamente atada a los habitantes de un lugar geográfico específico, sino que se produce también cuando no se tiene conocimiento de él. En la obra de Alice Munro, como en la de los grandes escritores, el sentido de localidad, de "conciencia visual" de un determinado entorno, se experimenta como palpable y real porque es una creación que nos afecta con la solidez y la particularidad de lo posible, estén o no presentes los detalles "documentales".

En Orlando, la novela de Virginia Woolf (1992:291), leemos:

The true length of a person's life, whatever the Dictionary of National Biography may say, is always a matter of dispute. For it is a difficult business -this time- keeping; nothing more quickly disorders it than contact with any of the arts [;]

Esta afirmación podría aplicarse también a nuestro sentido del espacio, ya que nuestra experiencia como lectores nos hace albergar en la memoria una gran variedad de ambientes y lugares que hemos experimentado como propios, y que se suman a los ya conocidos. Margaret Atwood (1994:130), escritora contemporánea a Alice Munro, también expone esta idea con una sola frase: "Put yourself into a different room, that's what the mind is for". Como lectores, a la vez que descubrimos un lugar, un ambiente que se desprende de un mundo imaginario, reconocemos que no nos es del todo extraño, que, simplemente, se nos ha revelado con palabras que desconocíamos.

Las primeras críticas publicadas sobre Munro se centraban en el análisis del tratamiento que la autora hace de unos escenarios rurales parecidos a los utilizados por Truman Capote o Carson McCullers, es decir, en la evocación de un sentido de localidad ("sense of place") y su influencia en las reacciones e impulsos de los personajes. Como ejemplo, podemos citar las conclusiones de Hugo McPherson en el libro

más valiosos que aquellas otras colecciones en las que se describen experiencias más urbanas de personajes desenraizados. Incluso en el influyente ensayo "Modernism Could Not Last Forever”, el crítico y escritor canadiense George Bowering (1980:4) se queja del realismo convencional que impera en la literatura canadiense, incluyendo a Munro en un grupo de buenos escritores que, a pesar de todo, "tell the normal realist story of sensitive child growing up to be disillusioned but maladjusted adult, the most personal proof of cause and effect." 
Literary History of Canada, editado por Carl F. Klink (1965), el prefacio de Hugh Garner a Dance of the Happy Shades (1968), el artículo “Alice Munro: A Portrait of the Artist" de Doug Spettigue (1969) y los ensayos de William New (1972), Frank Davey (1974), J. R. Struthers (1975), Hallvard Dahlie (1976), John Moss (1978), etc.

A partir de 1973, con el libro de Elizabeth Waterston titulado Survey: A Short Story of Canadian Literature (1973), se comienza a estudiar a Alice Munro desde el punto de vista de las particularidades de una literatura femenina y a relacionarla con recurrencias de perspectiva y temáticas ofrecidas por otras autoras canadienses como Margaret Laurence, Margaret Atwood, Mavis Gallant, Marian Engel o Audrey Thomas. Entre estos trabajos se encuentran los de William French (1975), Beverly J. Rasporich (1976), Dolores Gros-Louis (1976), Lorna Irvine (1986), etc.

Fue el artículo de William H. New (1976), "Pronouns and Propositions: Alice Munro's Stories”, el que abrió la posibilidad de un estudio textual de las narraciones de Munro, analizando estilísticamente el lenguaje de sus relatos, lenguaje que continuamente niega la posibilidad de la comunicación y la clasificación de las experiencias. Por otra parte, en el artículo "A Madman Loose in the World: The Vision of Alice Munro”, Rae M. Macdonald (1976) vislumbra esa otra realidad casi maligna que Munro muestra en sus relatos: la desfiguración de un mundo cotidiano y la dislocación que se manifiesta a través de un mundo grotesco que se nos aparece por entre las grietas de un entorno familiar aparentemente estable.

La dualidad de la visión de la obra de Alice Munro, centrada en los conceptos de lo cotidiano y lo extraño, es el principal foco de interés de una gran parte de la crítica en los años 80 y 90, que ha intentado analizar desde diferentes perspectivas las estrategias mediante las cuales la autora rompe los límites de la realidad empírica y se mueve más allá de un realismo convencional. Estas perspectivas se plasman en tres tendencias: la crítica feminista, el estudio de orientación narratológica (centrado en una doble visión a través de los narradores) y el estudio estilístico del lenguaje.

Podemos citar los libros de Louis Mackendrick (1983), de Coral Ann Howells (1987), W. R. Martin (1987), E. D. Blodgett (1988), Beverly J. Rasporich (1990), Magdalene Redekop (1992), Ajay Heble (1994), etc.

Con esta breve revisión de una parte de la crítica más relevante sobre Munro, no sólo he intentado proporcionar una bibliografía al interesado en esta autora, sino perfilar la orientación de mi análisis en este artículo. Mi intención es retomar el concepto de lugar (al que la crítica dio preferencia en un principio), pero no considerado como evocación o plasmación de una referencia externa, de un "lugar real" que la obra literaria representa, sino considerado como un principio organizativo emanado del propio texto que configura la progresión de la trama ${ }^{2}$. En el relato que analizaremos a conti-

2. El concepto de lugar, a cuyas implicaciones Kenneth Burke (1969) dedica un extenso análisis, aparece en esencia definido en torno a dos posibilidades: como un marco que contiene una acción y unos personajes, o como escenario causante de sus acciones. Nuestra intención es explorar qué otras implicaciones 
nuación, el concepto de lugar se presenta a través de un proceso metafórico creado por una conciencia central - la narradora en primera persona - mediante la transferencia de las propiedades de un paisaje a ciertos aspectos de la vida de un personaje en concreto, el padre de la narradora. Gracias a este proceso de identificación, el personaje principal, la narradora como niña, lleva a cabo un proceso de aprendizaje a través de sus contradictorias percepciones del espacio que le rodea.

El tipo de conocimiento del mundo que nos ofrecen muchos de los relatos iniciales de Alice Munro, y entre ellos "Walker Brothers Cowboy", se basa en una apropiación de la experiencia como "territorios visitados". En ellos, el tiempo se detiene y la identidad de los personajes se concibe gracias a la presentación de una situación estática que produce la "visualización" de un espacio como ilustración de un rasgo esencial de los personajes. Esta visualización de un espacio no es posible mediante la mera descripción inicial de un escenario que contiene a los personajes; se hace viable únicamente tras un proceso de análisis y evaluación, que da como resultado una percepción renovada que agrupa las características comunes de las personas y los objetos de un mundo conocido.

Nuestra apreciación del relato no consiste en adivinar las peculiaridades de un espacio que podría ser real, sino en comprender los conflictos en los que se ve envuelto el personaje a través de una toma de conciencia de las paradojas implicadas en la relación con su entorno geográfico. La interpretación de los estímulos visuales que realiza la protagonista - cuyo nombre no se menciona en el relato, al igual que el de su padre - se configura a la vez como una fuente de acceso a la realidad presente y conocida y como un obstáculo insalvable que le impide conocer el pasado de los seres que la rodean.

Después de una descripción de estos procesos, dirigiremos nuestra atención hacia otro aspecto relacionado con el espacio: la aparente cualidad documental que se desprende, en el relato, de la observación detallada de los aspectos que rodean a los personajes. Munro incluye en "Walker Brothers Cowboy" - como en muchos otros de sus relatos, aunque a través de diferentes procedimientos - aquellos aspectos que son seguros, reconocibles y predecibles de una realidad cotidiana, para añadirles una carga oculta de irrealidad, sorpresa o incertidumbre. En este relato existen estrategias que no permiten al lector contemplar el escenario en el que se desenvuelven los personajes como una realidad estable y permanente, ya que este mismo concepto de estabilidad se convierte en una hipótesis desechable sobre la realidad. De este modo, desde una aten-

puede albergar este concepto con respecto a nuestra noción de argumento además de las sugeridas por Kenneth Burke (1969:3-20), Mieke Bal (1985:93), Helmut Bonheim (1986:27) o Seymour Chatman (1990:109-133). Nuestro análisis no se relaciona con la denominada "crítica espacial" representada por el trabajo de Joseph Frank (1963). En este tipo de crítica, el término "espacial” se utiliza en un sentido figurado para denominar una forma de aproximación a determinados textos modernistas y postmodernistas que anulan la importancia de la progresión temporal en nuestra asimilación de la obra. 
ción al concepto espacial, mi análisis se relaciona con las tendencias más recientes en el estudio de la producción de Munro, que han dejado atrás el énfasis temático para profundizar en el estudio de los procedimientos textuales a través de los cuales se crean o se eluden significados en las obras.

Hemos mencionado anteriormente que en "Walker Brothers Cowboy" asimilamos el concepto de espacio a través de un proceso metafórico. Esta afirmación no significa que el relato esté basado en una sucesión de metáforas que acerquen al texto a un tipo de prosa "cuasi-poética", sino que se pueden establecer ciertos paralelismos entre el modo de operar de la metáfora y las estrategias textuales que nos ayudan a asimilar la significación de este relato. El tropo de la metáfora surge al poner en relación dos o más palabras cuyos significados aparentemente pertenecen a esferas muy diferentes de contenido. Existe un significante (una palabra) que rechaza su significado habitual para albergar otras connotaciones inusuales ${ }^{3}$. Según Jonathan Culler (1983:194), el escritor, a través de este proceso, descubre una "verdad"; efectúa una profundización sobre una emoción o una experiencia que surge de la identificación de la cualidad común de dos objetos o sensaciones:

Truth will not begin until the moment when the writer takes two different objects, sets down the relation between them that is the analogue in the world of art to the unique relation of the law of causation in the world of the sciences, and locks them together in the rings of beautiful style, or even, when, like life itself, in bringing together two sensations with a common quality he extracts their essence by uniting them with one another to withdraw them from the contingencies of time $[\ldots]^{4}$

La metáfora forja nuevos lazos entre aspectos de la realidad y descubre nuevos parecidos, de modo que, en este sentido, es el tropo más representativo del lenguaje poético porque resume las semejanzas u opiniones cualitativas tal como las revela nuestra sensibilidad. Del mismo modo, crea un enigma lingüístico e interpretativo y apela a nuestros sentidos, emociones o intuición para verificar su validez. Después de este proceso de aprendizaje lingüístico, nuestros modelos para clasificar la experiencia aparecen revitalizados. Gracias a un sensación de sorpresa inicial, producida al tener

Nuestro artículo tampoco tiene como objetivo explorar las implicaciones del regionalismo en el relato de Munro, aunque a este respecto, los ensayos de George Woodcock (1987:21-43), Robert Kroetsch (1989) y (1995) o Margaret Atwood (1996:25-45) proporcionan útiles introducciones al estudio de la literatura regionalista en Canadá.

3. Hemos tomado estos dos términos del estudio de Jean Cohen (1974:27), que ha su vez son tomados del Cours de Linguistique Générale de Ferdinand de Saussure. Cohen (1974:104-133) proporciona una ilustrativa explicación de la función de los recursos poéticos y de su interpretación por parte del lector. Véase además, la definición de la metáfora de Heinrich Lausberg (1976:181), Bice Mortara Garavelli (1988:163) y Genevieve Lloyd (1993:59).

4. Jonathan Culler utiliza este pasaje tomado de A la Reserche du Temps Perdu (1954:889) para ilustrar su concepción de la metáfora. 
contacto con una expresión que parece incongruente o extraña, se destruyen relaciones que creíamos asentadas (entre el significado y el significante) y una palabra o un concepto, en el proceso final de la lectura, presenta una serie de asociaciones diferentes a las habituales. Esta es la labor del poeta, como ya la había definido Viktor Shklovski (1970:137-8):

El poeta retira todos los carteles de su lugar; es el instigador de la revolución de los objetos. En los poetas los objetos se revelan, rechazan sus antiguos nombres y se cargan de un sentido suplementario con un nombre nuevo. El poeta se sirve de imágenes, de tropos, para hacer comparaciones: al fuego lo llama por ejemplo flor roja o aplica un nuevo epíteto a la palabra antigua, o bien dice como Baudelaire, que la carroña tenía sus piernas al aire como una mujer lúbrica. El poeta realiza un desplazamiento semántico, aparta la noción de la serie semántica donde se encontraba y la ubica con la ayuda de otras palabras (tropos) en otra serie semántica. Percibimos así la novedad, la inserción del objeto en una nueva serie. La nueva palabra se adosa al objeto como una nueva envoltura; se retira el cartel. (Enfasis añadido)

Estableciendo el paralelismo anteriormente mencionado entre este procedimiento de comprensión retórico y el desarrollo del relato "Walker Brothers Cowboy", podemos decir que éste se construye sobre la base de un cambio de sentido: una situación establecida como habitual o ordinaria al comienzo del relato - confirmada inicialmente en la narración a través de unas relaciones de cotidianeidad entre un personaje y su entorno espacial- se verá alterada por una interpretación nueva provocada por un elemento extraño o ajeno a la existencia anterior. Finalmente, una serie de asociaciones nuevas suplantarán a un conocimiento del mundo que se daba por sentado ${ }^{5}$. Lo esencial para nuestra hipótesis es que este proceso de "reinterpretación" que experimenta el personaje central se basa en las manifestaciones cambiantes del espacio. El conocimiento del mundo que nos proporciona la narradora se canaliza a través de un proceso de identificación entre dos conceptos que pertenecen a diferentes esferas: las personas y los paisajes.

Al final de este proceso de identificación, el conjunto de aspectos físicos, tangibles y conocidos del mundo de la protagonista rechazan su significado habitual para albergar otro contenido; es decir, el significante, el "soporte sólido" de un significado, se ha transformado para dar otro sentido a la narración. Para la niña protagonista, este "soporte sólido" aparece representado, primero, por la figura de su padre y, segundo,

5. Viktor Shklovski (1970:127-141) en su ensayo titulado "La Construcción de la 'Nouvelle’ y de la Novela" estudia la forma en que algunos relatos breves desarrollan figuras literarias o retóricas como el retruécano, el contraste o los paralelismos a través de argumentos basados en las confusiones, el desciframiento de enigmas o los dobles desenlaces. Mi estudio parte de una hipótesis parecida, aunque presta atención a otros componentes del relato, y estudia las analogías entre el proceso de comprensión de la protagonista del relato y la metáfora -tropo que Shklovski no trata. 
por los parajes conocidos cercanos a su casa. Veamos a continuación cómo estas ideas aparecen plasmadas en el texto.

El relato comienza con una invitación. El padre de la protagonista invita a ésta hacer una visita al lago, un lugar que aparentemente resulta familiar para todos los miembros de la familia. La frase inicial del relato - "After supper my father says 'want to go down and see if the Lake's still there?"'- es muy significativa, ya que la narración comienza con una pregunta, que a pesar de tener una intención humorística, cuestiona la solidez o la permanencia de un espacio. Es el padre, por lo tanto, quien introduce esta falta de certeza, esta falta de aserción que, como apreciamos más tarde a medida que leemos el relato, se convierte en el punto de partida para introducir la posibilidad de que el mundo que conocen los personajes es más inseguro e inestable de lo que puede parecer a primera vista.

Para la protagonista, su padre representa desde el primer momento el atractivo de aquellos ámbitos que están más allá del aburrido mundo de la casa y de las tareas que allí tiene que desempeñar por ser una niña. El padre es el personaje que acapara la atención de la niña, y para ella significa aventura, movimiento. Como reflejo de esta idea, el relato se compone de dos excursiones que la niña realiza con él: la visita al lago y el recorrido que efectúa en su habitual ronda como vendedor ambulante. De ahí el irónico título del relato, "Walker Brothers Cowboy", que hace mención a la actividad del padre, el cual, a pesar de ser presentado como un héroe errante, es en realidad un representante venido a menos contratado por la firma Walker Brothers.

En estos paseos, la niña va registrando rápidamente los lugares por los que pasan en su recorrido hacia el lago. Sin embargo, al llegar, no obtenemos del texto ninguna descripción del lago, ni ninguna referencia sensorial que nos anuncie que padre e hija se encuentran allí y lo están viendo. En cambio, la narradora incluye la explicación de su padre, que le intenta hacer comprender cómo es la parte del mundo en la que viven:

He tells me how the Great Lakes came to be. All where Lake Huron is now, he says, used to be flat land, a wide flat plain. Then came the ice, creeping down from the north, pushing deep into the low places. Like that -and he shows me his hand with his spread fingers pressing the rock-hard ground where we are sitting. His fingers make hardly any impression at all and he says, "Well, the old ice cap had a lot more power behind it than this hand has". And then the ice went back, shrank back towards the North Pole where it came from, and left its fingers of ice in the deep places it had gouged, and ice turned to lakes and there they were today. They were new as time went. (p.3)

Cuando la niña escucha las explicaciones de su padre, rechaza la posibilidad de que hayan existido otros paisajes donde ahora se encuentra éste, le asusta la idea de que el lago no haya estado siempre allí. No reconoce su conexión con unos orígenes comunes que puedan compartirse en forma de tiempo y de historia. A continuación, y dentro del mismo párrafo, la niña piensa: 
I try to see that plain before me, dinosaurs walking on it, but I am not able even to imagine the shore of the Lake when the Indians were there, before Tuppertown. The tiny share we have of time appals me, though my father seems to regard it with tranquillity. Even my father, who sometimes seems to have been at home in the world as long as it has lasted, has really lived on this earth only a little longer than I have, in terms of all the time there has been to live in. He has not known a time, any more than I, when automobiles and electric lights did not at least exist. He was not alive when this century started. I will be barely alive - old, old -when it ends. (p.3)

Este intento de describir un mundo "objetivo", un escenario común dado a priori y compartido por los habitantes de un mismo país, no consigue producir un lazo de referencialidad que conecte a la niña con un mundo exterior. Para ella, la descripción de su padre le parece una hipótesis amenazante; no puede asimilar la inmensidad cambiante de la naturaleza y del tiempo y opta por quedarse con una idea reconfortante de los lugares tal como ella los ha conocido siempre. El párrafo continúa:

I do not like to think of it. I wish the Lake to be always just a lake, with the safeswimming floats marking it, and the breakwater and the lights of Tuppertown.

y así la niña expresa su deseo de que su futuro se acomode a las reglas de lo que ha conocido siempre6.

Con esta primera presentación del paisaje y de las interpretaciones que de él hacen los personajes, la narradora nos está preparando para comprender las claves del núcleo del relato. Cuanto más nos adentramos en la narración, vamos descubriendo que el personaje del padre se configura como el centro de gravedad del relato porque alrededor de él giran las apreciaciones de la narradora. Su personalidad se nos presenta a través de una identificación con aquellos lugares que ha de recorrer al salir de casa diariamente, con la carretera que recorre en su destartalado coche por su "territorio", un distrito que le es asignado por su compañía. Posee un lenguaje especial para adular a las damas, y se adentra en las casas de sus clientes sin que sus hijos estén autorizados a seguirle:

How much this kind of farmhouse, this kind of afternoon, seem to me to belong to that one decade in time, just as my father's hat does, his bright flared tie, our car with its wide running board (an Essex, and long past its prime). Cars somewhat like it, many older, none dustier, sit in the farmyards. Some are past running and have their doors pulled off, their seats removed for use on porches. No living things to be seen, chickens or cattle. Except dogs. There are dogs, lying in any kind of shade they can

6. La misma Munro ha declarado en alguna ocasión que sus relatos tratan precisamente de las formas de pensar sobre el mundo que la gente utiliza para poder seguir viviendo: "The ways people discover for getting through life" (Geoff Hancock, 1982:108). 
find, dreaming, their lean sides rising and sinking rapidly. They get up when my father opens the car door, he has to speak to them. "Nice boy, there's a boy, nice old boy." They quiet down, go back to their shade. He should know how to quiet animals, he has held desperate foxes with tongs around their necks. One gentling voice for the dogs and another, rousing, cheerful for calling at doors. "Hello there Missus, it's the Walker Brothers man and what are you out of today?" A door opens, he disappears. Forbidden to follow, forbidden even to leave the car, we can just wait and wonder what he says. (p. 8)

Con su sentido del humor, inalterable ante cualquier humillación, y sus improvisadas canciones, el padre convierte las carreteras polvorientas y las solitarias casas, cerradas, sucias y hostiles en un mundo lleno de magia para la niña. Ella absorbe con avidez todos estos aspectos y los asocia a su carácter. Sin embargo, durante este paseo, el factor sorpresa se va haciendo poco a poco posible, y el relato se concentrará finalmente en un nuevo elemento: su padre abandona su territorio habitual para visitar a una antigua novia. Entonces aparece un personaje desconocido, Nora, una jovial y rolliza mujer soltera que vive con su madre ciega. Nora revela a la niña la existencia de una parte de la vida de su padre que ella no conocía. Su padre y "esa mujer" mantienen una conversación llena de bromas y alusiones privadas que la niña no llega a comprender del todo. Nora y su padre bailan, beben whisky, coquetean. Cuando acaba esta visita, en el párrafo que cierra el relato, leemos:

So my father drives and my brother watches the road for rabbits and I feel my father's life flowing back from our car in the last of the afternoon, darkening and turning strange, like a landscape that has an enchanment on it, making it kindly, ordinary and familiar while you are looking at it, but changing it, once your back is turned, into something you will never know, with all kinds of weathers, and distances you cannot imagine. (p.18)

Al final del relato, esa carretera que cruza una tierra llana y desolada se convierte en un lugar real, "asimilable" por la mente de la narradora. Si le impresiona como un espacio real no es porque ha aparecido descrito en detalle, sino porque representa y refleja una penetrante percepción de la vida de su padre que le muestra otro lado de su personalidad. La asimilación de un espacio va unida a una revelación sobre un aspecto oculto de un ser conocido. El personaje del padre se ha transformado en paisaje y el paisaje en un proceso cambiante, como la personalidad humana. Su padre ha adoptado las mismas cualidades cambiantes del paisaje que ella se negaba a admitir en un principio, cuando lo acompaña al lago. En su padre también hay distancias, lugares, cambios que ella nunca podrá conocer, como las fases anteriores a la aparición del lago.

Esta unión de características pertenecientes a distintos componentes de la narración (un personaje y varios lugares) es la actividad perceptual que finalmente 
se instaura en la mente del lector como el incentivo que proporciona movimiento y proyección temática a la trama. Las superficies de los objetos que la niña comprendía como hechos estables e incuestionables se vuelven inalcanzables cuando ella intenta asimilarlos en su totalidad, cuando intenta reconciliar todas sus múltiples manifestaciones. Así, su padre se convierte en un paisaje "darkening and turning strange" y se produce esa dualidad de visión entre dos dimensiones de la realidad que muchos críticos especializados en la produccion de Munro han denominado "the familiar and the strange" 7.

Por esta razón, la figura metafórica que se utiliza en el último párrafo del relato "my father's life flowing back [...] like a landscape that has an enchanment" no sólo se utiliza para materializar una percepción inusitada (como ilustración de un punto de observación concreto), sino que ilumina también el proceso temporal de la trama. No es un procedimiento periférico o aislado, sino que funciona como la visualización del conflicto presentado. Como en el proceso básico de comprensión de una metáfora, en este relato se produce una desviación del código cotidiano y se da un paso hacia un terreno desconocido, hacia una realidad dominada por reglas diferentes y extrañas que finalmente demuestran ser más verdaderas. Una serie de asociaciones suplantará a otra en la mente de la protagonista desde ese momento. La esencia de la realidad cotidiana es subversiva, como parece comprender la narradora, porque evidencia la poca solidez de lo que ella creía "su mundo". Este conocimiento supone inquietud e incertidumbre y conlleva una nueva interpretación, una nueva adecuación a un mundo que se muestra diferente.

Si recordamos en este punto las palabras de Shklovski (1970:137-8) citadas más arriba sobre el desplazamiento de un objeto fuera de su entorno ordinario, quizá podamos afirmar ahora con respecto a la estructura de "Walker Brothers Cowboy" que al personaje del padre se le ha "colocado" fuera de la serie de asociaciones habituales, creándose, así, una "revolución" en un esquema asentado de conocimiento de la protagonista.

Sin embargo, esta revolución, esta toma de conciencia, no produce una revelación o epifanía (Pickering, 1989:49) propiamente dicha, no ilumina el pasado de su padre; simplemente corrobora la existencia de dimensiones de su vida que la niña no podrá conocer nunca. La vida de su padre se le antoja ahora como un misterio fuera de su alcance, similar a los misterios del tiempo y del espacio a los que ella había sido "iniciada" al comienzo del relato. Durante esa inesperada visita, la narradora logra a duras penas vislumbrar un pasado que nunca le será accesible o intuir una historia para

7. W. R. Martin (1987:1-13) dedica un capítulo entero a este aspecto temático y Catherine Sheldrick Ross (1983:114-6) señala que, en los primeros relatos de Munro, la rutina se funde ocasionalmente con un mundo encantado o lleno de locura, "the familiar landscapeof the lakeshore, which is later revealed in an unfamiliar aspect". 
siempre silenciada, que en su mente permanecerá llena de indeterminación y especulaciones.

Por esta razón, la revelación en este relato se podría relacionar con las conclusiones de Thomas M. Leitch (1989:133) con respecto a ciertos tipos de relato en los que no se ofrece al lector una revelación satisfactoria porque no descubren la naturaleza de las cosas, sino que desvelan que la verdadera naturaleza de las cosas nunca podrá ser revelada; los relatos destruyen la creencia en la existencia de identidades estables. En los relatos que Leitch menciona, "The Capital of the World" y "A Clean, Well-Lighted Place", de Ernest Hemingway, el lector no pasa de la ignorancia al conocimiento, sino de un falsa sensación de certidumbre a una auténtica sensación de incertidumbre. Los personajes de estos relatos:

undergo experiences which do not so much enlighten audiences by means of authoritative revelations as disabuse them of their illusions about the world the story presents and represents without substituting any positive or more comprehensive wisdom.

Por otra parte, y por lo que respecta a la estructura de la narración, podemos observar que ésta no consiste en una secuencia de acontecimientos o en un cambio en la situación de los personajes, sino en un ajuste de pensamiento o de comprensión de un personaje central que observa. Este personaje, que es narrador a la vez, no actúa, sino que su papel es el de observador y sus percepciones se asimilan como diferentes fases que hacen avanzar a la trama. Como otros muchos relatos que no pueden ser considerados como un recuento objetivado de una secuencia de sucesos que no están sujetos a la modificación del carácter de uno o más personajes, "Walker Brothers Cowboy" nos hace reconsiderar nuestra noción de lo que hemos de entender por "argumento" y preguntarnos cuáles son los elementos narrativos mínimos necesarios para formar una historia.

A principios del siglo veinte, y especialmente a partir de modernismo, se produce un cambio en la noción de "argumento". Las convenciones del siglo diecinueve, presentadas como modelo de experiencia reorganizable y clasificable por un narrador todopoderoso, son rechazadas. Este narrador, que dotaba a la narración de un principio y final claros, aparece reemplazado por una multitud de perspectivas fieles al tiempo subjetivo en el que cada personaje está inmerso ${ }^{8}$. Según A. A. Mendilow (1952:48), una de las consecuencias de esta evolución de la novela:

8. A. A. Mendilow (1952) estudia de qué forma las técnicas de la ficción modernista estaban implicadas en una nueva concepción del tiempo influida por la teoría de la relatividad. Como uno de los manifiestos fundamentales de la época, véase la conferencia de Virginia Woolf "Mr. Bennet y Mrs. Brown" (1924) publicada en Hoffman \& Murphy (1988). 
is to be found in the replacing of causality on the plane of action by pure sequence on the plane of thought-feeling - a kind of picaresque novel of the day-dreaming mind.

Se concede mucha más importancia a los pensamientos de los personajes que a sus actos. La clara línea que une el principio y el fin de una trama convencional se desvirtúa, prestándose cada vez más atención a cómo los personajes conciben su pasado o sus relaciones con otros personajes. El concepto de trama queda reducido a una noción temporal: la fórmula "personajes en un tiempo" se confirma como el requisito mínimo para la creación de un relato completo y satisfactorio como tal ${ }^{9}$. Sin embargo, pueden surgir problemas con relación a la validez de esta fórmula. John Gerlach (1989:74) propone una reflexión sobre esta cuestión:

When do we finally reach the minimum unit of story, that is, a free-standing, complete and satisfying prose fiction? Might a story even become something else -a poem, for instance? If that is posible, do such discriminations ultimately matter? Are we likely to look at a work differently if it is, or is not, a story?

Gerlach examina las implicaciones de esta pregunta comparando algunos relatos breves con otros casos de obras narrativas breves cuya clasificación es problemática, como el poema en prosa, el "sketch" descriptivo, el mero recuento de un incidente intrascendente, etc. Su análisis comienza con el caso de una "pieza" en prosa en la que se narra una excursión de dos niños con su padre en busca de un lago helado donde poder patinar. Esta narración se detiene en un momento en el que los niños miran a través del hielo. Entonces Gerlach (1989:80) se pregunta: "Isn't looking at fish through ice also an event?". Este caso le hace recapacitar sobre su definición de lo que es un "suceso" y sobre los elementos mínimos necesarios para crear un relato literario, que al comienzo de su ensayo quedaba definido como un conflicto desarrollado en el tiempo. Finalmente concluye que la noción de relato breve no se debe hacer depender de lo que realmente "ocurre" en la narración, sino de las especulaciones que el texto crea en el lector:

a story is an invitation to contruct explanation, explanations about causality, connections, motives. [...] Plot is not necessary, nor is a fleshed-out sense of character and setting, as long as the reader is prodded to think in terms of character and motive.

9. Véanse las definiciones del concepto de trama basadas en un criterio temporal de Gerald Prince (Gerlach, 1989:75), Jan Miel (1969:917), Meir Sternberg (1978:19), Paul Ricoeur (1987:131), Mieke Bal (1985:5), Seymour Chatman (1990:45), R. S. Crane (1988:135) o Arthur Honeywell (1988:240). Si se busca un resumen crítico sobre la función del tiempo en el estudio de la literatura, véase William Harmon (1977:127-138). 
Un relato se crea, por lo tanto, cuando nos hace pensar en personas inmersas en un tiempo y en un espacio, independientemente de que contenga acción o no, cambios o conflictos dramáticos.

Podemos comparar el relato "Walker Brothers Cowboy" con el relato que Gerlach utiliza como ejemplo, titulado "In Search of a Clear View", para confirmar que la actitud del personaje resumida en la enunciación "looking at my father" o "looking at the lake" también es un suceso. Una simple percepción conlleva suficiente magnitud temática para que nos satisfaga como una historia. Este relato, concediendo prominencia a una relación entre un sujeto y un paisaje, lleva a primer plano la noción de que no se puede establecer a priori qué tipo de sucesos forman una trama - por su peso dramático o su transcendencia -, ya que ésta se forma cuando el lector toma conciencia de un conjunto de relaciones sugeridas por el texto. Son precisamente las estrategias textuales las que modifican e incluso invierten nuestra jerarquía convencional de relevancia, nuestra preconcepción de qué elementos han de considerarse importantes; un detalle o un incidente aparentemente trivial puede ser dotado contextualmente de gran significación. La percepción, es decir, el contacto real o imaginario de un sujeto y un objeto es criterio suficiente para que un relato sea completo.

Un punto de percepción con respecto a un objeto crea una perspectiva dentro del relato, y el concepto de perspectiva, además de poder considerarse como una estrategia que mediatiza la información presentada, puede concebirse como un suceso en sí mismo, como una decisión -relacionada con un personaje- sobre dónde y cómo posicionarse ante la realidad. Especialmente cuando nos encontramos con un narrador adulto que relata las experiencias de un periodo anterior de su vida, sus percepciones se convierten en una selección de qué tipo de realidad vivida ha decidido incluir en su narración. Como lectores, nuestro sentido de haber presenciado una historia y comprendido todos sus matices se produce cuando llegamos a ser conscientes de esta "decisión", que es la proyección de una conciencia (la de la narradora en primera persona, en el caso de "Walker Brothers Cowboy") que ha favorecido ciertos "caminos" para poner en comunicación los diferentes componentes del mundo imaginario de la obra.

Alice Munro, autora que en muchos aspectos presenta semejanzas con la obra de Proust, hace de la contemplación el argumento y el tema de la narración. Tal como afirma Genevieve Lloyd (1993:145), A La Reserche du Temps Perdu evoluciona a través de "a perceptual activity of the character contemplating: discoveries, shifts in distance and perspective, errors, connections, enthusiasms or dissapointments."

Algunas narraciones se organizan en torno a la presentación de un recorrido biográfico más o menos extenso (una red de múltiples relaciones entre los personajes, la resolución de un conflicto o de una insatisfacción inicial, un complejo de situaciones cambiantes, etc.). Otras veces no siguen la línea de una historia más o menos convencional, sino que se ciñen a una o varias conciencias centrales y su significado gira en torno a las condiciones especiales de percepción de estas conciencias. Los relatos se 
configuran entonces como análisis, como estudios de la personalidad, es decir, el narrador no se "mueve" con el personaje sino alrededor de él10. Este es el caso de "Walker Brothers Cowboy", donde el concepto de espacio no queda relegado a una mera descripción, entendida como un telón de fondo que proporciona una referencia visual para la presentación de una acción o un conflicto; en este caso, el espacio es el elemento que da un sentido de progresión al argumento.

Nuestra conciencia de un lugar se estimula en este relato, no tanto gracias a la descripción, sino a través de la revelación. Cuando la narradora llega a una conclusión, cuando obtiene un conocimiento verdadero sobre los seres que la rodean, este punto de percepción se asocia estrechamente con el lugar en el que ha llegado a esa deducción (como en el párrafo final de "Walker Brothers Cowboy" citado anteriormente).

La ausencia de una entidad narradora omnisciente que pueda corroborar (a través de un pacto ficticio con el lector) la existencia de lugares y objetos, favorece una mayor atención por parte del lector a la cambiante actividad perceptual mediadora del relato. No se puede afirmar que en "Walker Brothers Cowboy" los lugares tienen unas propiedades "reconocibles" o "predecibles", porque éstos sólo pueden concebirse a posteriori, después de que la narradora haya contemplado un segundo nivel de realidad que se le aparece más allá de sus conocidas superficies ${ }^{11}$.

El conflicto central de este relato se basa en el modo en que los individuos obtenemos conocimiento del mundo y de los demás, y Munro presenta este problema a través de unas percepciones ancladas en los detalles particulares de un espacio. Pero este acercamiento a la realidad se presenta como problemático; los pasajes que contienen descripciones convencionales son siempre engañosos porque en ellos se cuestiona su propia razón de ser. La categoría de la descripción, según Helmut Bonheim (1986:24), "includes the mere assertion that an object or condition exists". En contraposición a esta definición, Munro siempre incluye una atmósfera de irrealidad que amenaza con convertir a los objetos o a los lugares en presencias imaginadas, llenas de elementos

10. La narradora de "Walker Brothers Cowboy" no intenta recordar o transmitir una historia sino analizar ciertos aspectos de su pasado. Como resultado, la narración aparece dividida en secciones en las que se centra su actividad especulativa. Las implicaciones del tipo de organización textual denominada "espacial" en la literatura moderna han sido estudiadas por David Mickelsen (1981) o Jerome Klinkowitz (1981).

11. Coral Ann Howells (1987:17) analiza este mismo proceso, pero a través de la mezcla de códigos realistas y fantásticos:

Munro's and Laurence's heroines are brought up in such borderland territory and they retain their doubleness of vision in their adult lives. Their perceptions that the wilderness as place/state of mind is not something that can be entirely shut out are written into their stories, resulting in those moments of instability where cracks open in the realistic surface to reveal dark secret places within social enclosures. 
ilógicos. Así ocurre en la descripción de una de las granjas típicas cercanas a la casa de la narradora:

The land is flat, scorched, empty. Bush lots at the back of the farms hold shade, black pine-shade like pools nobody can ever get to. We bump up a long lane and at the end of it what could look more unwelcoming, more deserted than the tall unpainted farmhouse with grass growing uncut right up to the front door, green blinds down and a door upstairs opening on nothing but air? Many houses have this door, and I have never been able to find out why. I ask my father and he says they are for walking in your sleep. What? Well if you happen to be walking in your sleep and you want to step outside. (pp. 7-8). (Énfasis añadido)

Es de nuevo el padre quien introduce la posibilidad de que los objetos puedan tener una cualidad ilusoria, inventada, como esa puerta que se abre a la nada, cuya función es permitir el paso a una persona sonámbula. En este pasaje, el concepto de realidad del mundo, entendido como una referencia visual compartida por los personajes, es anulado al introducir un elemento de distorsión que transporta lo descrito a la esfera de lo imaginario, fantasmagórico o evanescente. Sólo tenemos que recordar la pregunta que abre el relato; el hecho de que sea una broma no impide que se produzca una impresión de inseguridad o desasosiego al contemplar un componente ilógico implícito en los espacios que rodean a los personajes.

Incluso cuando nos encontramos ante descripciones que aparentemente encajarían dentro de la noción de una descripción convencional, se produce en algún punto una disparidad de interpretaciones. La niña comprende desde un primer momento que no puede existir un solo lugar de referencia si hay más de una persona observando. Cuando los dos hermanos esperan a su padre en el coche mientras él intenta vender sus artículos, comienza una descripción:

One yard after another, then, the old cars, the pumps, dogs, views of grey barns and falling-down sheds and unturning windmills. The men, if they are working in the fields, are not in any fields that we can see. The children are far away, following dry creek beds or looking for strawberries, or else they are hidden in the house, spying at us through cracks in the blinds. The car seat has grown slick with our sweat. [...] We play I Spy, but it is hard to find many colours. Grey for the barns and sheds and toilets and houses, brown for the yard and fields, black or brown for the dogs. (p. 9)

En este punto del párrafo esta descripción cambia su función. Leemos a continuación:

The rusting cars show rainbow patches, in which I strain to pick out purple or green; likewise I peer at doors for shreds of old peeling paint, maroon or yellow. We can't play with letters, which would be better, because my brother is too young to 
spell. The game disintegrates away. He claims my colours are not fair, and wants extra turns. (p.9)

La niña ve colores que su hermano pequeño piensa que no existen realmente. El hecho de que los objetos tengan múltiples identidades y de que éstas no sean percibidas por todos los individuos que "miran" hace que la hipótesis de la referencia a un mundo externo sea algo imposible de compartir, incluso como una premisa de la que se hacen partícipes los mismos personajes. En este pasaje del texto se han contrastado dos tipos diferentes de realidad, la realidad empírica y la imaginada. La realidad empírica, fiel a un mundo externo, aparece como fraudulenta ante la realidad del descubrimiento y la síntesis personal.

Hemos comentado anteriormente que los personajes de "Walker Brothers Cowboy" no se conocen en el tiempo, sino en el espacio. Sus vínculos emocionales con personas y lugares abandonan la dimensión temporal y así, estos personajes aprenden a través de una reflexión sobre las cualidades de un entorno espacial. Podemos citar aquí las palabras de Gastón Bachelard (1969:8), que ilustran esta idea:

all we know is a sequence of fixations in the spaces of the being's stability, a being who does not want to melt away, and who, even in the past, when he sets out in search of things past, wants time to 'suspend' its flight. In its countless alveoli space contains compressed time. That is what space if for.

Sin embargo, este acercamiento al mundo se presenta como frustrante en "Walker Brothers Cowboy", ya que hace que el tiempo sólo se pueda asimilar como el contraste entre dos o más situaciones. Observamos que las vidas de los personajes no se articulan secuencialmente y, por lo tanto, desconocemos los procesos de su formación. Los cambios o las alteraciones en la vida de los personajes, o las modificaciones del conocimiento que unos tienen de otros no se presentan como una culminación (ya que no ha habido una transición que nos ayude a comprender dos visiones tan diferentes de un mismo personaje), sino que parecen ser el producto de una fuerza arbitraria, manifestaciones fuera de toda lógica o predicción. Este relato no nos ofrece un proceso de desarrollo de una o varias identidades, sino la posibilidad de una identidad múltiple. Los diferentes rasgos que encarnan los personajes no aparecen apoyados en una línea de coherencia cronológica o causa-efecto, sino que están disgregados, y la narradora se concentra en cada uno de ellos por separado, como dibujando por turnos secciones de un mismo paisaje.

Gracias a este predominio de una concepción espacial de la existencia, la experiencia no se expresa como cadena o secuencia, sino como concentración en aspectos, en fracciones independientes que no aparecen unidas en un sistema total de vida. Otros muchos relatos de Alice Munro también se componen de un estímulo o de una idea que va modificándose a medida que una narradora en primera persona reflexiona 
sobre ella, como "The Peace of Utrecht", "Something I've Been Meaning to Tell You", "The Spanish Lady", etc. Munro no "sigue" a ninguno de sus personajes continuadamente a través del curso total del relato. Al lector se le confronta con dos imágenes de los personajes, inmóviles en un momento de visión, como si fueran fotografías tomadas en momentos diferentes. De este modo, el personaje aparece representado como si formara parte de los objetos que lo rodean, partícipe de una unión visual y anímica irrepetible e inmutable como en una fotografía ${ }^{12}$.

Con estas estrategias, Alice Munro da nueva vida a una de las ideas básicas del relato breve, tal como la define Shklovski (1970:136): cada uno de nosotros tiene una existencia doble. "Walker Brothers Cowboy" también explota esa constante del género: esa doble relación con respecto a un mismo objeto, con respecto a una misma realidad. El final del relato ilustra esta cualidad subversiva e inquietante; al no existir un punto final concluyente, ni una sola realidad, el personaje y el lector quedan desamparados. El concepto de una realidad unívoca es cuestionado, está lleno de aspectos irreconciliables. Helen Hoy (1991:18) destaca en su artículo la cualidad caótica e indefinida de los finales de los relatos de Munro, que dejan constancia de que la falta de resolución de las cosas puede convertirse en permanente. El mundo cotidiano de hechos, detalles y objetos se revela de repente como una construcción ficcional de la narradora. En este relato, el espacio no es simplemente un escenario o una proyección que funciona como un eco de los estados anímicos de los personajes; se convierte en un concepto organizativo en la mente de la narradora, ya que su proceso de transferencia de propiedades entre personajes y paisajes proporciona un sentido de progresión al relato y crea el conflicto de la trama.

\section{Bibliografía}

ATwood, Margaret. 1994 (1983). Bones and Murder. London: Virago.

Atwood, Margaret. 1996 (1972). Survival. A Thematic Guide to Canadian Literature. Toronto: McClelland \& Stewart.

BAL, Mieke.1985. Narratology: Introduction to the Theory of Narrative. Toronto: University of Toronto Press.

Bachelard, Gastón. 1969 (1957). The Poetics of Space. Boston: Beacon Press.

12. Lorraine York (1988) proporciona un estudio de la obra de Munro desde el punto de vista de las relaciones entre el texto literario y la fotografía. Esta autora reconoce en la obra de Munro un manifiesto artístico diferente del de aquellos "fotógrafos ficcionales" de ciudades pequeñas como el escritor norteamericano Sherwood Anderson. Según York (1988:39-40), Munro representaría en sus relatos la paradoja de lo grotesco y lo hermoso en el retrato de personajes; no sólo revela y desenmascara la realidad, sino que la celebra y la crea, haciéndola más compleja, "adding delicate shadows to stark reality". 
BonHeIM, Helmut. 1986 (1982). The Narrative Modes: Techniques of the Short Story. Suffolk: D. S. Brewer.

Bowering, George. 1980. "Modernism Could Not Last Forever". Canadian Fiction Magazine, 32-33: 4-9.

Blodgett, E. D. 1988. Alice Munro. Boston: G. k. Hall \& Co.

BURKE, Kenneth. 1969 (1945). A Grammar of Motives. Berkeley: University of California Press.

Chatman, Seymour. 1990 (1978). Historia y Discurso: La Estructura Narrativa en la Novela y en el Cine. Madrid: Taurus Humanidades.

CoHEn, Jean. 1974 (1966). Estructura del Lenguaje Poético. Madrid: Gredos.

Crane, R. S. 1988. "The Concept of Plot". En Hoffman, Michael \& Murphy, Patrick. Eds. Essentials of the Theory of Fiction. Durham and London: Duke University Press. 131-142.

Culler, Jonathan. 1983 (1981). The Pursuit of Signs. Semiotics, Literature, Deconstruction. London: Routledge and Kegan Paul.

DAHLIE, Hallvard. 1976 (1972). “Munro, Alice”. En Vinson, James. Ed. Contemporary Novelists. New York: St. Martin's. 910-920.

DAVEy, Frank. 1974. “Alice Munro”. En From Here to Eternity: A Guide to English Canadian Literature Since 1960. Vol. II. Erin: Porcépic. 201-214.

FRANK, Joseph. 1968 (1963). The Widening Gyre. Crisis and Mastery in Modern Literature. Bloomington: Indiana University Press.

FrENCH, William. 1975. "The Women in Our Literary Life". Imperial Oil Review, 59, $1: 2-6$.

GARAVElli, Bice Mortara. 1988. Manual de Retórica. Madrid: Cátedra.

GARner, Hugh. Ed. "Foreword”. Dance of the Happy Shades. Toronto: Ryerson. vii-ix.

Gerlach, John. 1989. "The Margins of Narrative: The Very Short Story, The Prose Poem, and the Lyric". En Lohafer, susan \& Clarey, Jo Ellyn. Eds. Short Story Theory at a Crossroads. Baton Rouge and London: Louisiana University Press. 74-84.

Gros-Louis, Dolores. 1976. "Pen and Needles: Daughters and Mothers in Recent Canadian Literature". The Kate Chopin Newsletter, 2, 3: 8-15.

Hancock, Geoff. 1982. "An Interview with Alice Munro". Canadian Fiction Magazine, 43: 74-114.

Harmon, William. 1977. Time in Ezra Pound's Work. Chapel Hill: The University of North Carolina Press.

Heble, Ajay. 1994. The Tumble of Reason: Alice Munro's Discourse of Absence. Toronto: University of Toronto Press.

Honey Well, Arthur. 1988. "Plot in the Modern Novel”. En Hoffman \& Murphy. Eds. 238-250. 
ENTRE NUESTROS OJOS Y EL MUNDO: ASIMILACIÓN DEL CONCEPTO DE ESPACIO EN EL RELATO...

Howells, Coral Ann. 1987. Private and Fictional Worlds. Canadian Women Novelists of the 1970's. London: Methuen.

Hoy, Helen. 1991. “Alice Munro: 'Unforgettable, Indigestible Messages'”. Journal of Canadian Studies, 26, 5: 5-21.

IRVINE, Lorna. 1986. Sub/Version. Toronto: ECW Press.

KLINKOwITZ, Jerome. 1981. "The Novel as Artifact: Spatial Form in Contemporary Fiction”. En Smitten, Jeffrey R. \& Daghistany, Ann. Eds. Spatial Fom in Narrative. Ithaca and London: Cornell University press. 37-47.

Kroetsch, Robert. 1989. The Lovely Treachery of Words. Toronto: Oxford University Press.

KroEtsch, Robert. 1995. "Unhiding the Hidden”. En Ashcroft, Bill, Griffiths, Gareth \& Tiffin, Hellen. Eds. The Post-Colonial Studies Reader. London: Routledge. 394-397.

LAUSBERG, Heinrich. 1976 (1960). Manual de Retórica Literaria. Madrid: Gredos.

LEITCH, Thomas M. 1989. "The Debunking Rhythm of the American Short Story". En Lohafer, Susan \& Clarey, Jo Ellyn. Eds. 130-147.

Lloyd, Genevieve. 1993. Being in Time. Selves and Narrators in Philosophy and Literature. London and New York: Routledge.

MacDonald, Rae McCarthy. 1976. “A Madman Loose in the World: The Vision of Alice Munro". Modern Fiction Studies, 22, Fall: 365-74.

MacKendrick, Louis K. Ed. 1983. Probable Fictions. Alice Munro's Narrative Acts. Downsview: ECW Press.

Martin, W. R. 1987. Alice Munro. Paradox and Parallel. Alberta: The University of Alberta Press.

McPherson, Hugo. 1965. "Fiction 1940-1960”. En Klinck, Carl F. Ed. Literary History of Canada: Canadian Literature in English. Toronto: University of Toronto Press. 720-722.

Mendilow, A. A. 1952. Time and the Novel. Holland: Ysel Press.

Mickelsen, David. 1981. "Types of Spatial Structure in Narrative". En Smitten \& Daghistany. Eds. 63-78.

MIEL, Jan. 1969. "Temporal Form in the Novel”. MLN, 84: 916-930.

Moss, John. Ed. 1978. Here and Now: A Critical Anthology of the Canadian Novel. Vol. I. Toronto: N.C. 7-13

Munro, Alice. 1982. "What Is Real?". The Canadian Forum. Sept.: 5-36.

Munro, Alice. 1983 (1968). "Walker Brothers Cowboy". Dance of the Happy Shades. Harmondsworth: Penguin. 1-18.

Munro, Alice. 1983. "The Peace of Utrecht”. Dance of the Happy Shades. 190-210.

Munro, Alice. 1985 (1974). "Something I've Been Meaning To Tell You”. Something I've Been Meaning To Tell You. Harmondsworth: Penguin. 9-29.

Munro, Alice. 1985 (1974).“The Spanish Lady”. Something I've Been Meaning To Tell You. 170-184. 
NEw, W. H. 1972. “The Canadian Short Story: Introduction". World Literature Written in English, II, April: 4-25.

New, W. H. 1976. "Pronouns and Propositions: Alice Munro's Stories". Open Letter, 3, 5: 40-49.

Pickering, Jean. 1989. "Time and the Short Story”. En Hanson, Clare. Ed. ReReading the Short Story. London: MacMillan. 45-54.

RASPORICH, Beverly J. 1976. "Child-Women and Primitives in the Fiction of Alice Munro". Atlantis (Acadia University), I, Spring: 4-14.

Rasporich, Beverly J. 1990. Dance of the Sexes. Art and Gender in the Fiction of Alice Munro. Alberta: The University of Alberta Press.

RedeKoP, Magdalene. 1992. Mothers and Other Clowns. The Stories of Alice Munro. London: Routledge.

Ricoeur, Paul. 1987. Tiempo y Narración II: Configuración del Tiempo en el Relato de Ficción. Madrid: Ediciones Cristiandad.

Ross, Catherine Sheldrick. 1983. “'At Least Part Legend': The Fiction of Alice Munro”. En Mackendrick, L. K. Ed. 112-126.

ShKLOVSKI, Viktor. 1970 (1919). "La Construcción de la 'Nouvelle' y de la Novela”. En Todorov, Tzvetan. Ed. Teoría de la Literatura de los Formalistas Rusos. Buenos Aires: Signos. 127-157.

Spettigue, Doug. 1969. “Alice Munro: A Portrait of the Artist”. Alumni Gazette. University of Western Ontario, 45, 3: 3-27.

Sternberg, Meir. 1978. Expositional Modes and Temporal Ordering in Fiction. London: The Johns Hopkins University Press.

Stouck, David. 1988 (1984). Major Canadian Authors. Lincoln: University of Nebraska Press.

Struthers, J. R. 1975. "Alice Munro and the American South". Canadian Review of American Studies, 6, Fall: 196-204.

Waterston, Elizabeth. 1973. Survey: A Short History of Canadian Literature. Toronto: Methuen.

WoodCCOK, George. 1987. Northern Spring. The Flowering of Canadian Literature. Vancouver: Douglas \&McIntyre.

Woolf, Virginia. 1988 (1924). “Mr. Bennet and Mrs. Brown”. En Hoffman \& Murphy. Eds. 25-39.

Woolf, Virginia. 1992 (1928). Orlando. A Biography. Oxford: Oxford University Press.

YoRK, Lorraine. 1988. The Other Side of Dailiness: Photography in the Works of Alice Munro, Timothy Findley, Michael Ondaatje, Margaret Laurence. Toronto: ECW Press. 